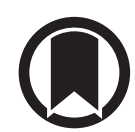

CrossMark

\section{In vivo demonstration of pulmonary microvascular involvement in COVID-19 using dual-energy computed tomography}

\author{
To the Editor:
}

Vascular involvement in coronavirus disease 2019 (COVID-19) has been suggested by several observations, such as the high rate of pulmonary embolism [1], the prothrombotic state and the proinflammatory biological profile $[2,3]$, as well as the pathological findings of severe endothelial injury and diffuse thrombosis [4]. Dual-energy computed tomography (DECT) allows specific imaging of the iodinated contrast agent distribution within the lung, which has been demonstrated as a surrogate marker of lung perfusion [5, 6]. Therefore, our goal was to assess in vivo pulmonary microvascular involvement in patients with COVID-19 pneumonia by using DECT and to determine whether vascular changes vary during the course of the disease.

This monocentric study was approved by our institutional ethics committee. We performed a retrospective analysis of patients hospitalised for COVID-19 pneumonia (confirmed by RT-PCR for severe acute respiratory syndrome coronavirus 2) who underwent enhanced DECT for clinical worsening of symptoms and/or hypoxaemia in search of pulmonary embolism.

Lung computed tomography (CT) angiography data were acquired on a dual-layer DE CT system (iQon*; Philips Healthcare) using a bolus-tracking technique with a threshold of $110 \mathrm{HU}$ in the main pulmonary artery enabling iodine image reconstruction, and reviewed by a senior radiologist and a senior pulmonologist. Predominant lung lesions such as ground-glass opacities (GGO) and alveolar consolidation, as well as their volumetric extension per lobe (0: none; $1: 0-25 \% ; 2: 26-50 \%$; $3: 51-75 \% ; 4: 76-100 \%)$ were rated. Presence and aspect of perfusion abnormalities were evaluated per lobe as an increase or decrease of perfusion blood volume (PBV) compared to the remote parenchyma. Lobar PBV was indexed to iodine concentration in the pulmonary trunk, by calculating the ratio of mean iodine concentration in a lobe to that in the pulmonary trunk. As lung perfusion may depend on the injection time, iodine concentrations ratios in the main pulmonary artery over the left atrium were recorded and expressed as median (interquartile range).

PBV distribution was tested for normality using d'Agostino-Pearson test. Unpaired t-test was used for PBV comparisons between lobes with predominant GGO and consolidation. A multiple linear regression was used to compare the lobar PBV between parenchymal type lesions as a function of the extension. Statistical significance was set at $\mathrm{p}<0.05$.

Five patients were included between 15 March and 30 April, 2020. All of them were male and median age was 70 years (range $45-88$ years). No patient had a history of chronic respiratory disease. Three patients $(60 \%)$ were admitted to the intensive care unit and one patient $(20 \%)$ died of acute respiratory failure. At the time of DECT imaging, four patients $(80 \%)$ had oxygen supplementation therapy (oxygen saturation $<90 \%$ on room air), two patients $(40 \%)$ had prophylactic anticoagulation therapy, while the remaining three $(60 \%)$ had long-term therapeutic anticoagulant doses. No patient had evidence of bacterial infection. Concurrently, D-dimer levels were $3107 \pm 3053 \mu \mathrm{g} \cdot \mathrm{L}^{-1}$, fibrinogen levels were $6.5 \pm 1.6 \mathrm{~g} \cdot \mathrm{L}^{-1}$, C-reactive protein levels were $93 \pm 45 \mathrm{mg} \cdot \mathrm{L}^{-1}$ and platelet count was $390000 \pm 234000 \mathrm{~mm}^{-3}$.

@ERSpublications

In COVID-19 pneumonia, dual-energy computed tomography has shown two patterns of lung perfusion along the course of the disease: an increase in perfusion blood volume (PBV) with groundglass opacities and a decrease in PBV with consolidation https://bit.ly/2Zh0XcI

Cite this article as: Si-Mohamed S, Chebib N, Sigovan M, et al. In vivo demonstration of pulmonary microvascular involvement in COVID-19 using dual-energy computed tomography. Eur Respir J 2020; 56 : 2002608 [https://doi.org/10.1183/13993003.02608-2020]. 

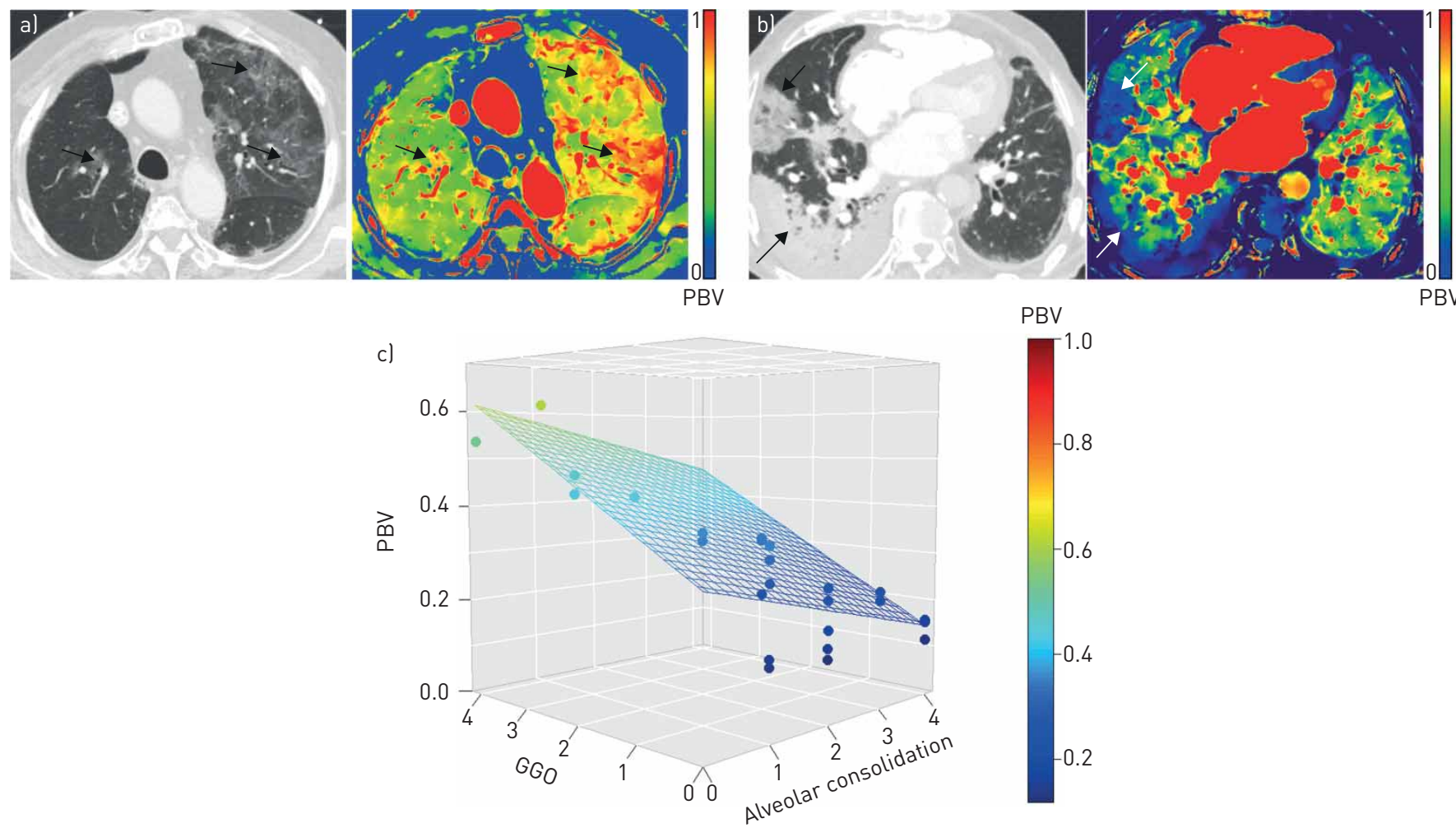

FIGURE 1 Key imaging features of microvascular involvement in coronavirus disease 2019 (COVID-19) using lung angiography dual-energy computed tomography scans. Perfusion blood volume (PBV) is defined as the ratio between iodine concentration in a pulmonary lobe and iodine concentration in the main pulmonary artery. a) Representative imaging features of the early clinical phase: increased PBV is seen (black arrows, right panel), matching with ground-glass opacities (GGO) (black arrows, left panel), relative to the areas without GGO. b) Representative imaging features of the late clinical phase: decreased PBV is seen (white arrows, right panel), matching with alveolar consolidation (black arrows, left panel), relative to the areas without consolidation. c) Three-dimensional graph of lobar PBV as a function of the presence and extension of GGO and alveolar consolidation. Each dot corresponds to a lobe, the coordinates of which are represented by PBV in the $z$-axis, GGO extension in $x$-axis and consolidation extension in $y$-axis (volumetric extension score, 0: none; 1: 0-25\%; 2: 26-50\%; 3: 51-75\%; 4: 76-100\%). Colour grid represents the multiple linear regression plane.

DECT was performed during the first week from the onset of symptoms in two patients (day 7 and 8 ) and after 2 weeks in three patients (day 17 and 18). No patient had pulmonary embolism. Patients in the early clinical phase had predominant GGO lesions (figure 1a) while patients in the late clinical phase had predominant consolidation (figure $1 \mathrm{~b}$ ). Median ratio of iodine concentration between main pulmonary artery and left atrium were calculated at 1.4 (1.3-3.1). Perfusion abnormalities were found in all lobes matching with corresponding parenchymal lesions. Mean \pm SD PBV was $0.48 \pm 0.09$ in lobes with predominant GGO and $0.22 \pm 0.08$ in lobes with predominant consolidation $(\mathrm{p}<0.0001)$. No perfusion abnormalities were found in the areas surrounding the lesions nor in the normal parenchymal areas. Multiple linear regression analysis demonstrated a significant correlation between PBV and parenchymal lesions $(\mathrm{R}=0.84, \mathrm{p}<0.0001$ ), with a positive coefficient between PBV and GGO (Pearson's $\mathrm{r}=0.83$ ) and a negative coefficient between PBV and consolidation (Pearson's $r=-0.51$ ) (figure 1c).

This pilot study has demonstrated that DECT may be used to assess pulmonary vascular involvement in vivo in patients with COVID-19 pneumonia. Two different patterns of lung perfusion were observed.

In the early clinical phase occurring in the first week since the onset of symptoms, the predominant parenchymal lesions were diffuse bilateral GGO and were associated with increased lung perfusion in the corresponding lobes. This observation suggests that hypoxaemia in these patients may be due to a ventilation/perfusion mismatch causing pulmonary shunting. We speculate that low ventilation/perfusion ratio may be related to decreased ventilation secondary to viral pneumonia, along with normal or increased perfusion which might be due to the loss of the physiological hypoxic vasoconstriction provoked by inflammatory cytokines. These vascular changes may correspond to the histopathological findings of intussusceptive angiogenesis found in a recent autopsy study [4].

During the later phase of the disease, occurring after 2 weeks, the predominant parenchymal lesions were bilateral alveolar consolidation and were associated with decreased lung perfusion in the affected lobes. 
The presence of lung hypoperfusion in the absence of detectable pulmonary embolism is a distinctive hallmark of COVID-19 and could be due to the endothelial dysfunction and the release of prothrombotic cytokines, often referred to as the "cytokine storm" and potentially leading to acute respiratory distress syndrome. These vascular changes are corroborated by pathological findings of endothelial dysfunction, diffuse coagulopathy affecting small vessels, and the formation of microthrombi [4]. All patients had increased inflammatory and prothrombotic biomarkers. It is also noteworthy that hypoperfusion lesions occurred despite anticoagulant therapy. Of note, description of pulmonary vascular manifestations of COVID-19 pneumonia using DECT has been previously reported [7, 8]. Radiological findings were mosaic perfusion patterns, vessel enlargement within and outside of lung opacities, and as peripheral perfusion defects with surrounding halos of increased perfusion $[7,8]$. These results are to a certain extent consistent with our findings. However, our study provides new insights on chronological changes in the pulmonary perfusion along the course of the disease and in relation to parenchymal CT features $[9,10]$.

There are limitations to our study, including the small number of patients and the retrospective design. Despite the predominant opacification of the pulmonary arteries of the DECT scans, the potential participation of a systemic arterial supply within areas of pneumonia cannot be ruled out. There was no sequential DECT study carried out in the same patient. However, CT scans were conducted for real life clinical purposes, taking into consideration renal and radiation risks.

In conclusion, DECT imaging demonstrated pulmonary microvascular involvement in COVID-19 pneumonia with two distinctive patterns. It may be used to better understand COVID-19 pathophysiology and herald new targets for therapeutic trials.

Salim Si-Mohamed $\oplus^{1,2}$, Nader Chebib ${ }^{3}$, Monica Sigovan ${ }^{2}$, Lea Zumbihl ${ }^{1,4}$, Ségoléne Turquier ${ }^{3}$, Sara Boccalini ${ }^{1,2}$, Loic Boussel $^{1,2}$, Jean-Francois Mornex ${ }^{3,4,5}$, Vincent Cottin $\oplus^{3,4,5}$ and Philippe Douek ${ }^{1,2}$

${ }^{1}$ Radiology Dept, Hospices Civils de Lyon, CHU Louis Pradel, Lyon, France. ${ }^{2}$ Claude Bernard Lyon 1 University, INSALyon, UJM-Saint Etienne, CNRS, Inserm, CREATIS UMR 5220, U1206, Lyon, France. ${ }^{3}$ Pneumology Dept, Hospices Civils de Lyon, National Reference Center for Rare Diseases, Lyon, France. ${ }^{4}$ Claude Bernard Lyon 1 University, University of Lyon, INRA, UMR754, Lyon, France. ${ }^{5}$ Pulmonary Diseases, Competence Center for severe pulmonary hypertension, Lyon, France.

Correspondence: Salim Si-Mohamed, CHU Louis Pradel, Département d'imagerie cardiovasculaire, 59 Boulevard Pinel, 69500 Bron, France. E-mail: salim.si-mohamed@chu-lyon.fr

Received: 2 July 2020 | Accepted after revision: 2 Sept 2020

Acknowledgements: We thank Valerie Leitman (University of Lyon) for contributions to manuscript editing, and Adeline Mansuy (Hospices civils de Lyon) for the research proposal submission.

Conflict of interest: S. Si-Mohamed reports personal fees for consultancy from Boehringer Ingelheim and non-financial support for meeting attendance from Guerbet, outside the submitted work. N. Chebib reports non-financial support for meeting attendance from CSL Behring, Actelion, GSK, Boehringer Ingelheim, Roche and MSD, outside the submitted work. M. Sigovan has nothing to disclose. L. Zumbihl has nothing to disclose. S. Turquier has nothing to disclose. S. Boccalini has nothing to disclose. L. Boussel has nothing to disclose. J-F. Mornex reports grants, personal fees and non-financial support from CSL Behring and LFB, personal fees and non-financial support from Actelion, personal fees from Roche, Boehringer Ingelheim, GSK, Novartis, Chiesi and Elivie, outside the submitted work. V. Cottin reports personal fees for advisory board work and lectures, and non-financial support for meeting attendance from Actelion, grants, personal fees for consultancy and lectures, and non-financial support for meeting attendance from Boehringer Ingelheim, personal fees for advisory board and data monitoring committee work from Bayer/MSD and Galapagos, personal fees for advisory board work and lectures from Novartis, personal fees for data monitoring and steering committee work, consultancy and lectures, and non-financial support for meeting attendance from Roche/Promedior, personal fees for lectures from Sanofi and AstraZeneca, personal fees for data monitoring committee work from Celgene and Galecto, personal fees for advisory board work from Shionogi, personal fees for steering committee work from Fibrogen, outside the submitted work. P. Douek has nothing to disclose.

\section{References}

1 Poyiadji N, Cormier P, Patel PY, et al. Acute pulmonary embolism and COVID-19. Radiology 2020; in press [https://doi.org/10.1148/radiol.2020201955].

2 Huang C, Wang Y, Li X, et al. Clinical features of patients infected with 2019 novel coronavirus in Wuhan, China. Lancet 2020; 395: 497-506.

3 Zhou F, Yu T, Du R, et al. Clinical course and risk factors for mortality of adult inpatients with COVID-19 in Wuhan, China: a retrospective cohort study. Lancet 2020; 395: 1054-1062.

4 Ackermann M, Verleden SE, Kuehnel $M$, et al. Pulmonary vascular endothelialitis, thrombosis, and angiogenesis in Covid-19. N Engl J Med 2020: 383: 120-128.

5 Fuld MK, Halaweish AF, Haynes SE, et al. Pulmonary perfused blood volume with dual-energy CT as surrogate for pulmonary perfusion assessed with dynamic multidetector CT. Radiology 2013; 267: 747-756.

6 Si-Mohamed S, Moreau-Triby C, Tylski P, et al. Head-to-head comparison of lung perfusion with dual-energy CT and SPECT-CT. Diagn Interv Imaging 2020; 101: 299-310. 
7 Lang M, Som A, Carey D, et al. Pulmonary vascular manifestations of COVID-19 pneumonia. Radiol Cardiothorac Imaging 2020; 2: e200277.

8 Lang M, Som A, Mendoza DP, et al. Hypoxaemia related to COVID-19: vascular and perfusion abnormalities on dual-energy CT. Lancet Infect Dis 2020; in press [https://doi.org/10.1016/S1473-3099(20)30367-4].

9 Shi H, Han X, Jiang N, et al. Radiological findings from 81 patients with COVID-19 pneumonia in Wuhan, China: a descriptive study. Lancet Infect Dis 2020; 20: 425-434.

10 Pan F, Ye T, Sun P, et al. Time course of lung changes at chest CT during recovery from coronavirus disease 2019 (COVID-19). Radiology 2020; 295: 715-721.

Copyright @eERS 2020 .

This version is distributed under the terms of the Creative Commons Attribution Non-Commercial Licence 4.0. 